\title{
Features of flame stabilization in near wall flows
}

\author{
Vladimir Lukashov*, and Vladimir Terekhov \\ Institute of Thermophysics SB RAS, 630090, Novosibirsk, 1 Lavrent'iev Ave., Russia
}

\begin{abstract}
The results of the experimental and numerical study of the influence of obstacle geometry on the conditions of flame stabilisation in the turbulent boundary layer with fuel injection near a porous surface are presented. The features of the detachment zones formation in the turbulent reacting flow behind the obstacle are studied. The obtained results are compared with the data on flame-off conditions when flowing over a flat surface.
\end{abstract}

\section{Introduction}

An active or passive impact on flow in the flame front edge can effectively control the combustion in the boundary layer. In particular, as it was shown in the works of E.P. Volchkov [1] when burning near a flat surface, through which the gaseous fuel was injected, the features of the flow formation played an important role. In the same paper, the regularities of the flame-off conditions were experimentally established, and the model was proposed. It was based on the diffusion combustion approximation, the boundary layer theory and the assumption that extinction occurs when the flame front reaches the wall. The range of conditions in which the combustion is stable near a flat surface can be significantly expanded by using the stabilizing devices. Traditionally, ribs or back steps of various geometries were used to form a recirculation zone with an extended residence time of initial reactants and reaction products mixture. The heat release and transverse momentum flow caused by injection of the fuel mixture can substantially change the regularities of this class of flows [2]. A very limited amount of works were devoted to the investigation of conditions of flame detachment in a separated flow near a flat surface with injection. In [35], based on visual observations, the diagrams of combustion and flame-off modes were obtained for some hydrocarbon fuels and the geometry of the barriers forming the detached flow zones. There are other ways to stabilize the flame. In particularly the detached rib may be one of the promising areas. The structure of the flow and heat transfer over a detached rib for non-reactive flows was numerically studied in [6]. It was shown that an increase in the gap between the rib and the surface leads to a change in the structure of the recirculation region. The regimes in which there was an increase in heat exchange with the wall were observed in comparison with the case of a rib placed on the surface.

In this paper, we have determined the conditions of flame-off at the detached rib located above the wall, while the main attention was paid to the peculiarities of the flow in the vicinity of the obstacle.

*Corresponding author: luka@itp.nsc.ru 


\section{Experimental Installation}
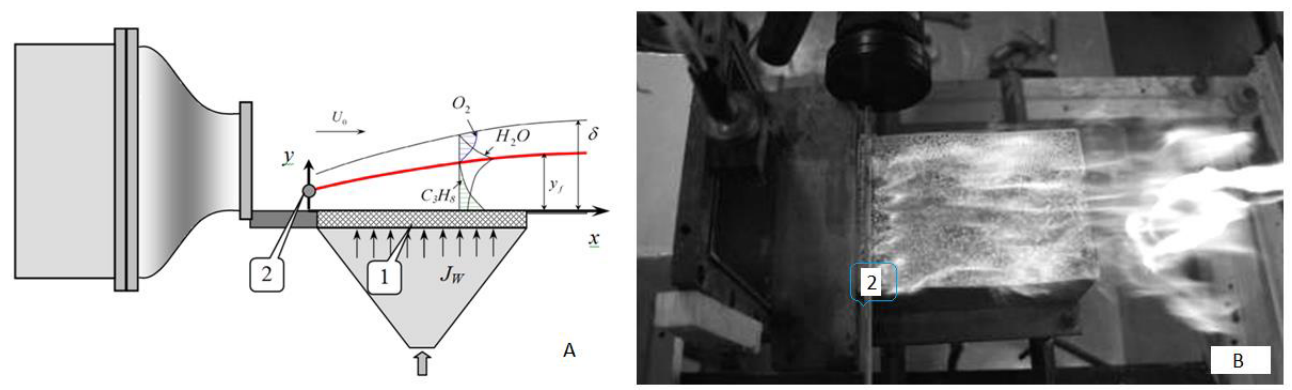

Fig. 1. The scheme of the working section of the unit for investigating the wall flow with injection and gas fuel combustion in the air stream (A). Photo (B) of the wall stream with propane injection and burning, top view.

The experimental studies were carried out in a subsonic wind tunnel with a channel section at the entrance to the working part $108 \times 108 \mathrm{~mm}$. The speed of air flow over the plate varied in the range $1 \ldots 20 \mathrm{~m} / \mathrm{s}$. The diagram of the working part is shown in Figure 1. The fuel was uniformly blown into the boundary layer through the lower horizontal porous plate (1) with a plan dimension $\mathrm{S}=95 \times 145 \mathrm{~mm}$. The injected fuel was propane (or methane) diluted with $\mathrm{CO} 2$. To prepare the fuel mixture, digital gas flow regulators were used. Immediately before the beginning of the porous section, a rib (2), being a ceramic cylinder $5 \mathrm{~mm}$ in diameter, was installed. The rib was located directly on the surface, or was fixed at a height of up to $3 \mathrm{~mm}$ above the wall (detached rib).

To measure the velocity distribution in the reacting flow the PIV method was used. The plane of the light sheet was oriented perpendicular to the permeable surface, which allowed obtaining data on a two-dimensional flow field (longitudinal and vertical velocity components). Because of the peculiarities of the method used to diagnose the dynamics of the wall flow, the measurements were carried out sequentially and statistically independently in overlapping regions of the space of $\sim 40 \times 40 \mathrm{~mm}$ in size. When using the optical method in the wall flow above the permeable wall, it is required to ensure a uniform dusting of the flow by light scattering particles. For this purpose, separate, independent dusting of $\mathrm{TiO} 2$ particles with both the main stream and the fuel mixture was used.

\section{Theoretical model}

Under the conditions of the experimental studies the numerical simulation was carried out. This allowed obtaining a more complete picture of the processes in the turbulent reactive separated flow in the boundary layer behind the barrier. In particular, the simulation allowed obtaining detailed information on the distribution of concentrations of all the mixture components, refining the data on the parameters in the immediate vicinity of the walls and identifying some other factors that were not directly measured. Numerical simulation in this paper was based on the URANS approach. We solved non-stationary averaged equations of Navier-Stokes, energy and concentrations. As a model of turbulence, the SST k-model was used, thermal and diffusion turbulent flows were determined according to the gradient model. The interaction of turbulent pulsations and combustion was modeled according to the concept of the EDC vortex dissipation. 


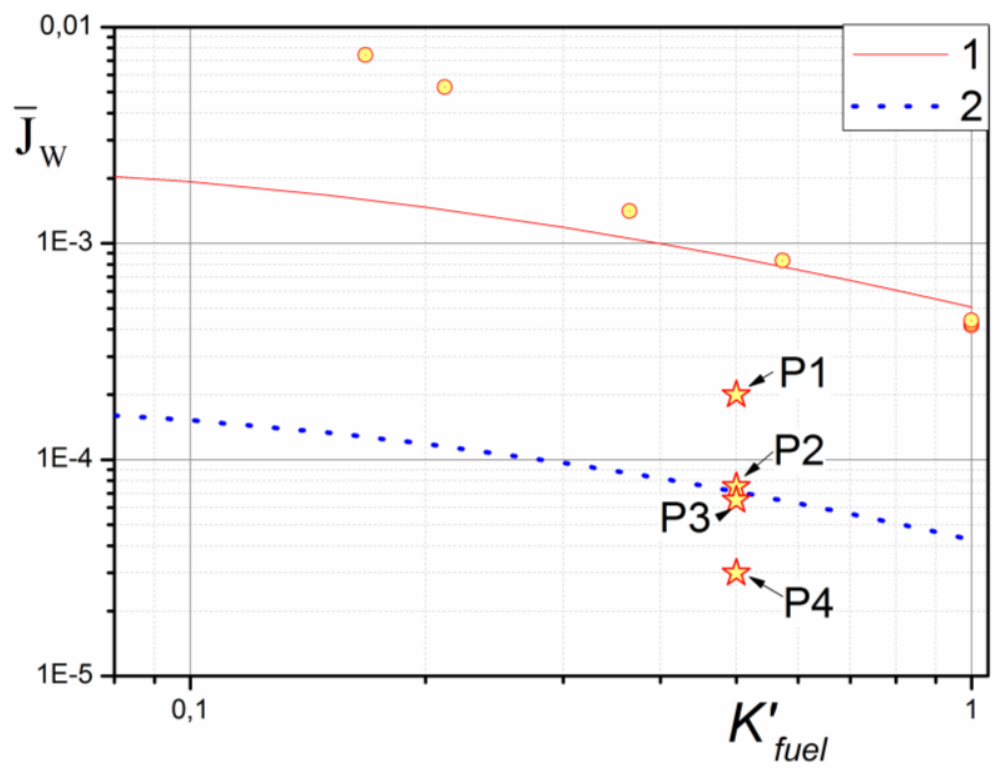

Fig. 2. Range of parameters of stable diffusion combustion in the boundary layer with the injection of the hydrocarbon mixture with $\mathrm{CO}_{2}$ (above the line). Lines: $1-\mathrm{CH}_{4}, 2-\mathrm{C}_{3} \mathrm{H}_{8}$. Figures - the experimental data of the authors for the $\mathrm{CH}_{4} / \mathrm{CO}_{2}$ fuel mixture, points P1...P4 simulation results for propane.

\section{Results and discussion}

In the diffusion combustion approximation in the boundary layer with injection of a hydrocarbon fuel mixture diluted with an inert diluent, there is a unique relationship [1] for a relative intensity of the injection $\bar{J}_{W}$; and $K_{\text {fuel }}^{1}$ is the mass fraction of the fuel component in the fuel mixture. The estimates for a number of hydrocarbons are shown in Figure 2. It is seen that at $K_{\text {fuel }}^{l}>0.4$, these boundaries adequately describe the experimental data. In order to expand the combustion area, it is necessary to use a flame stabilizer.

First of all one can note that in the boundary layer without a barrier only one stable combustion mode is observed [1] when the flame develops starting from a certain distance from the start of the field of injection. In the case of the detached flow in such regimes, a few attachment points were observed in addition to the previously described combustion regime in the boundary layer. At high blowing rates and high velocities of the incoming flow the flame can be stabilized in the separation zone behind the obstacle, and the front of the flame develops downstream the obstacle (P1 point on Figure 2). The second mode, in which the combustion was divided into two regions: directly behind the barrier in the recirculation zone and substantially lower in the flow in the boundary layer, was observed as the intensity of the injection was reduced (P2 point). With further reduction in fuel injection through the wall, a third regime was possible, in which combustion was observed only in the recirculation region behind the obstacle ( $\mathrm{P} 3$ point). In the conditions indicated as P4 the combustion became impossible. The detachment of the rib from the surface further reduces the minimum injection intensity sufficient to ensure combustion. This is due to the peculiarities of forming the detached wall flow, which significantly change if the rib is placed above the section of porous blowing. 

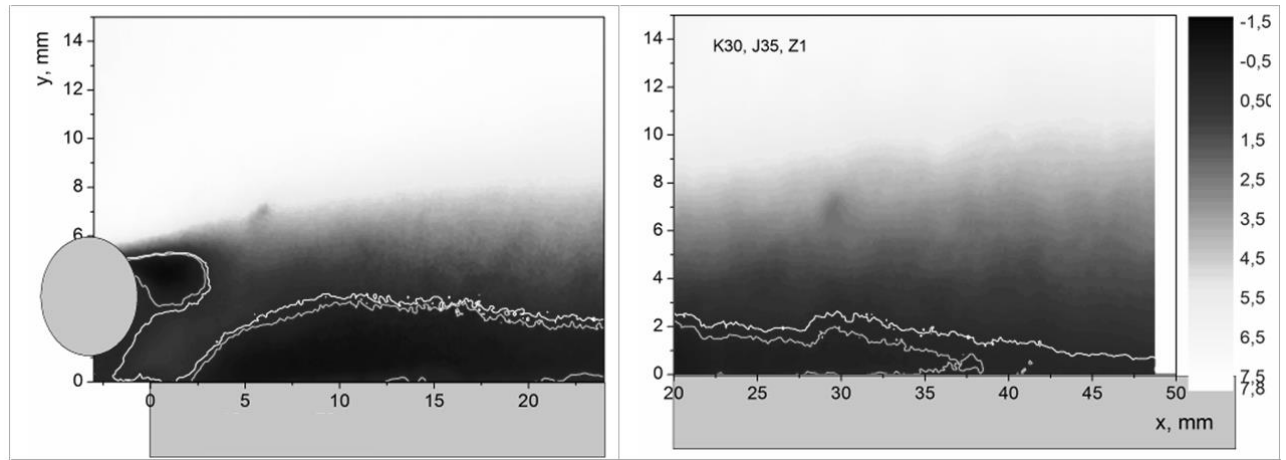

Fig.3. The measured longitudinal velocity component. The fuel mixture $\mathrm{C}_{3} \mathrm{H}_{8} / \mathrm{CO}_{2}$ with propane mass fraction $K^{1}{ }_{C 3 H 8}=0.3$, injection $\bar{J}_{W}=0.35 \%$, air flow velocity $U_{0}=7.5 \mathrm{~m} / \mathrm{s}$.

Figure 3 shows the results of measurements of the field of the longitudinal velocity component when the propane-air flame is stabilized behind the detached rib. It is evident that two regions of the recurrent flow are formed by isotachs $\pm 0.1 \mathrm{~m} / \mathrm{s}$ (light lines in the picture).

The visible flame leading edge in the section of the PIV system laser sheet plane was at 6-8 $\mathrm{mm}$ downstream from the surface of the detached rib. The formation of the region of the recirculating near wall flow is apparently determined by two factors: the heat release in the flame front and the transverse flow of matter specified by injection of the fuel mixture.

\section{Conclusion}

The results of the experimental and numerical study of the turbulent reacting flow behind a detached rib near a porous surface with fuel injected through the wall have been analyzed. In a separated flow, unlike a boundary layer on a flat surface, several combustion regimes may be observed. The diagram was obtained for the flame-off condition both for the case of a plane surface and for a surface with an obstacle above it, and a comparison was made with the model proposed by E. Volchkov [1].

This work was supported by the RFBR grant № 15-08-05662a.

\section{References}

1. E.P. Volchkov, V.V. Lukashov, V.V. Terekhov, K. Hanjalic, Comb. Flame $\mathbf{1 6 0}$ 1999 (2013)

2. V.V. Terekhov, V.I. Terekhov, J. Appl. Mech. Tech. Phys. 58, 254 (2017)

3. A. Ramachandra, B.N. Raghunandan, Comb. Sci.Techn. 36109 (1983)

4. B.N. Raghunandan, G.P. Yogesh, XXII Int. Symp. on Combust. 1501 (1988)

5. T.A. Rohmat, H. Katoh, T. Obara, T. Yoshihashi, S. Ohyagi, AIAA J. 36, 1945 (1998)

6. T.V. Bogatko, V.I. Terekhov, J. Appl. Mech. and Tech. Physics 56, 57 (2015) 